\title{
The Influence of Investor Sentiment on Monetary Policy Transmission
}

\author{
Xiaoqiu Liu, Yangqing Xiao ${ }^{\text {a, * }}$ \\ Finance and Economics College, Jimei University, Xiamen, Fujian Province, China \\ axyq@xmu.edu.cn \\ *Corresponding author: Yangqing Xiao
}

Keywords: monetary policy; investor sentiment; impulse response analysis; variance decomposition

\begin{abstract}
This paper studies the influence of investor sentiment on monetary policy transmission from the perspective of behavioral finance. Two VAR models are established in this experiment, while the factor of investor sentiment is contained in only one model. The dynamic relationships between variables in these two models are compared to determine whether the effect of monetary policy on macro economy can be influenced by investor sentiment. The empirical results show that investor sentiment can exacerbate price and output fluctuations caused by monetary policy. The explanatory power of monetary policy increases when investor sentiment is taken into consideration.
\end{abstract}

\section{Introduction}

The implementation effects of monetary policies is a research focus in the academic circle. Many scholars have conducted in-depth studies on this issue. Some researchers theoretically studied the different transmission mechanisms of monetary policy, while others empirically tested the transmission effects of monetary policies. It must be emphasized that the traditional financial theory holds that market participants are perfectly rational; the impact of monetary policies on the macro economy is analyzed on that premise. However, in recent years, the development of behavioral finance makes people realize that investors are not perfectly rational; they can be affected by various psychological factors and behave irrationally in the financial market. Until now, knowledge on behavioral finance has been widely applied in financial studies; many scholars have studied the impact of investor sentiment on the transmission of monetary policies. Relevant research can help government departments to accurately estimate the implementation effects and control the implementation time of monetary policies, and help investors as well as consumers to better predict the macroeconomic trends and adjust their investment as well as consumption decisions.

\section{Literature Review}

\subsection{The influence of investor sentiment on monetary policy transmission.}

Qi Luo constructed FAVAR models with and without emotional factors in 2015, and investigated the influence of emotional factors on monetary policy transmission by comparing the two models. [1] Empirical results show that in the model containing emotional factors, monetary policy has relatively good inhibition effects on economic overheating and can better explain the inflation. At the same time, the explanatory power of monetary policy on other macroeconomic variables is also increased.

Yi Zhou constructed SVAR models with and without emotional factors in 2011; the research conclusion is similar to that of Qi Luo. [2]

Alexander Kurov holds that monetary policy has significant impacts on investor sentiment, and that the impacts are different in bull and bear markets. [3] The impacts of monetary policy on the stock market are greater in a bear market, because at that time, the stock market is more sensitive to changes in investor sentiment.

Yun-yun Sui constructed two MS-VAR models in 2017, with one containing investor sentiment proxy variables, and the other excluding investor sentiment indicators. [4] The empirical result shows 
that investor sentiment does have an impact on the transmission of monetary policy; that effect shows asymmetry in upsurge and downturn periods of stock market.

\subsection{Measurement of investor sentiment.}

Investor sentiment proxy indicators can be divided into three categories. The first category is direct sentiment indicators. To collect this kind of data, the researcher needs to distribute questionnaires to different types of investors. Through the statistical analysis of survey results, the researcher can find investors' expectations on the development trend of the market and compile relevant indicators. At home, such indicators mainly include the BSI index of CCTV, Yale-CCER Chinese Investor Confidence Index and consumer confidence index. Consumer confidence index reflects the confidence of citizens on the country's economic conditions; it is a comprehensive index which can show consumers' views and expectations on interest rates, market prices as well as employment and income conditions.

The second category is indirect emotional indicators obtained from data of the stock market. Such indicators include trading volume, turnover rate, the number of new accounts, price-earnings ratio, IPO yield, closed-end fund discount rate and so on.

The third category refers to composite index. Through some processing methods, such as principal component analysis and Kalman filter method, a number of variables can be integrated into a single, comprehensive index of investor sentiment. The most representative composite index is the BW index constructed by Baker and Wurgler in 2006. Six indicators are involved in this index, namely the turnover rate, IPO number, IPO first day yield, closed-end fund discount rate, the proportion of new shares issued and dividends. Through the principal component analysis, BW index, a comprehensive index on investor sentiment, is constructed.

To sum up, some scholars have studied the influence of investor sentiment on monetary policy transmission from the perspective of behavioral finance; the effects of monetary policy on both macroeconomic environment and stock market have been analyzed. Some researchers have studied the asymmetric effect of monetary policy. The stock market is divided into bull market and bear market according to the linear time series model; the macro economy is divided into different stages according to the economic cycle theory. This paper uses VAR model to determine whether investor sentiment has an impact on the transmission effect of monetary policy by comparing the dynamic relationships between variables in models with and without investor sentiment factors.

\section{Index Selection and Data Source}

The ultimate goals of monetary policy are economic growth and price stability. Hence in this paper, output and price are selected as the proxy variables of macro-economy. Output can be measured by GDP, but the authority in our country only publishes quarterly GDP data at present. With reference to most scholars' practice, this paper replaces GDP with the growth rate of industrial added value year-on-year as the proxy index of output, and takes the growth rate of consumer price index (CPI) as the proxy index of price.

As for the index of money supply, M2 represents the money supply in broader sense; it includes both deposits and cash. Under the current economic development level of China, M2 can not only reflect citizens' purchasing power at present, but reflect their potential purchasing power in the future. Thus, this paper chooses M2 as the proxy variable of monetary policy.

As for the measurement of investor sentiment, there are many transmission mechanisms of monetary policy which involve all aspects of the economy. This paper needs a macro-investor sentiment indicator which can reflect the expectations of investors on the overall macroeconomic situation. The consumer confidence index (CCI) is selected.

Samples in this paper range for 120 months from January 2008 to December 2017. All indicators are monthly data obtained from the National Bureau of Statistics and the website of East Money. The data analysis software is Eviews 8.0. 


\section{Empirical Process and Analysis}

\subsection{Data processing and stationary test.}

This paper selects indicators of money supply (M2), consumer confidence index (CCI), consumer price index (CPI) year-on-year growth rate and industrial added value (Q) year-on-year growth rate. First, the classical Sensus X-12 method is used in the seasonal adjustments of M2 and CPI; then these two indicators are recorded as M2X and CPIX respectively. Then, in order to prevent multicollinearity, the logarithms of M2X and CCI are respectively recorded as $\operatorname{lnM} 2 \mathrm{X}$ and $\operatorname{lnCCI}$. In the empirical study, data with percentage signs were processed to remove the percentage signs.

The results of the traditional ADF unit root test show that all variables are first-order difference stationary at $1 \%$ significance level. Thus all variables are processed by first-order difference and recorded as dlnM2X, dlnCCI, dCPIX and dQ respectively.

\subsection{Estimation and test of VAR model.}

Estimation and test of VAR model with investor sentiment. The VAR model is established with four indexes: dlnM2X, dlnCCI, dCPIX and dQ. According to AIC criterion, the most suitable order is the fourth order. Therefore, the VAR (4) model is established. The model parameters are shown in Table 1.

Table 1. VAR (4) model parameters

\begin{tabular}{lllll}
\hline & DLNM2X & DCPIX & DQ & DLNCCI \\
\hline DLNM2X(-1) & 0.096613 & -13.08074 & -32.15249 & -0.514047 \\
DLNM2X(-2) & 0.25071 & 4.577813 & 74.92849 & 0.115213 \\
DLNM2X(-3) & 0.103811 & 16.00692 & -50.4894 & 0.003253 \\
DLNM2X(-4) & 0.039056 & -3.685755 & 84.18476 & -0.264177 \\
DCPIX(-1) & $6.71 E-05$ & 0.014706 & 1.720833 & 0.000705 \\
DCPIX(-2) & -0.001234 & 0.082625 & 1.134224 & 0.001983 \\
DCPIX(-3) & 0.000195 & 0.102516 & -1.217415 & -0.006778 \\
DCPIX(-4) & -0.001334 & 0.180758 & -0.312145 & 0.002827 \\
DQ(-1) & $-6.57 E-05$ & 0.106189 & -0.690692 & 0.000488 \\
DQ(-2) & -0.000492 & 0.077224 & -0.397129 & 0.002351 \\
DQ(-3) & -0.000106 & 0.055437 & -0.205723 & 0.001717 \\
DQ(-4) & 0.000231 & 0.022113 & 0.023761 & 0.001359 \\
DLNCCI(-1) & -0.039072 & -2.765365 & 1.923784 & -0.164803 \\
DLNCCI(-2) & 0.017893 & 0.59642 & 11.57951 & -0.120983 \\
DLNCCI(-3) & -0.025191 & 4.758773 & 10.6177 & 0.070486 \\
DLNCCI(-4) & -0.006763 & 0.65733 & -28.9967 & -0.197441 \\
C & 0.005794 & -0.057193 & -1.04846 & 0.009484 \\
\hline
\end{tabular}

Granger causality test shows that dlnM2X, dCPIX and dQX are not Granger causes of dlnCCI; dlnCCI is not Granger cause of dCPIX and dQX; dlnM2X and dQX are Granger causes of dCPIX; dlnM2X and dCPIX are Granger causes of dQX. The stability test shows that all roots of unity fall inside the unit circle, so the model is stable.

Estimation and test of VAR model without investor sentiment. The VAR model is established with three indexes: dlnM2X, dCPIX and dQ. According to AIC criterion, the most suitable order is the fourth order. Therefore, the VAR (4) model is established. Model parameters are shown in Table 2.

Granger causality test shows, dQX and dCPIX are each other's Granger reason; dlnM2X is not Granger reason of dCPIX; dlnM2X is Granger reason of dQX. The stability test shows that all roots of unity fall inside the unit circle, so the model is stable. 


\subsection{Impulse response analysis.}

Impulse response analysis examines the impacts of exogenous shocks on endogenous variables. The following two models are impacted by monetary policy variables to observe the response of price and output variables to monetary policy changes.

Impulse response analysis of VAR model with investor sentiment. Under the impact of a standard deviation of money supply (M2), CPI responds immediately to the disturbance. Response in the first phase is -0.06 , which is a negative value. Then the response rapidly decreases to zero and starts to increase positively, reaching 0.094 in the fourth phase. Then the response drops rapidly and turns negative, reaching -0.099 in the fifth phase. After that, it turns to a positive value again, reaching 0.128 in the sixth period. In the twentieth period, the response become steady and tends to zero.

Table 2. VAR (4) model parameters

\begin{tabular}{llll}
\hline & DLNM2X & DCPIX & DQ \\
\hline DLNM2X(-1) & 0.091792 & -11.15849 & -27.88439 \\
DLNM2X(-2) & 0.260549 & 3.207286 & 52.2724 \\
DLNM2X(-3) & 0.115909 & 13.55537 & -45.59257 \\
DLNM2X(-4) & 0.048728 & -4.127242 & 103.5128 \\
DCPIX(-1) & -0.00057 & 0.0032 & 1.36174 \\
DCPIX(-2) & -0.00076 & 0.05901 & 1.16823 \\
DCPIX(-3) & $1.75 E-05$ & 0.145327 & -0.818552 \\
DCPIX(-4) & -0.001247 & 0.18806 & -0.506464 \\
DQ(-1) & -0.000118 & 0.109261 & -0.711545 \\
DQ(-2) & -0.000461 & 0.080455 & -0.401161 \\
DQ(-3) & -0.000195 & 0.057978 & -0.195655 \\
DQ(-4) & 0.000225 & 0.016873 & -0.015343 \\
C & 0.005414 & -0.026466 & -1.1104 \\
\hline
\end{tabular}

$\mathrm{Q}$ also responds immediately to the disturbance of M2. Response in first phase is 0.32 . Then positive and negative responses occur alternately, with response value gradually increasing, reaching 1.29 in the fifth phase and -0.67 in the sixth phase. After that, although the response turns to positive, the response degrees are not significant. At the sixteenth phase, the response become steady and tends to zero.

Impulse response analysis of VAR model without investor sentiment. CPI responds immediately to the disturbance of M2. Response at the first phase was -0.06 , which is a negative value. Then the response decreases rapidly to zero and then increases positively, reaching 0.09 in the fourth phase. Then the value decreases rapidly and become negative, reaching -0.09 in the fifth phase, and then turns positive again, reaching 0.12 in the sixth phase. In the nineteenth period, the response become steady and tends to zero.

$\mathrm{Q}$ also responds immediately to the disturbance of $\mathrm{M} 2$. The positive and negative responses appear alternately. The response degree is 0.3 in the first phase and -0.5 in the second phase. Then the response degree increases gradually, reaching 1.2 in the fifth phase and -0.7 in the sixth phase. After that, although the response turns positive, the response degrees are small. At the sixteenth phase, the response become steady and tends to zero.

\subsection{Variance decomposition.}

Variance decomposition of model with investor sentiment. The variance decomposition including investor sentiment shows that the proportion of variable CPI that can be explained by M2 is $2 \%$ in the first three periods, and increases gradually from the fourth period, reaching $10 \%$ in the sixth period. After the tenth period the proportion reaches $11 \%$ and remains stable. The proportion of variable Q that can be explained by M2 slowly rises from 1\% in the first phase to $8 \%$ in the fourth phase, and then rapidly rises to $20 \%$ in the sixth phase. Afterwards, the value stabilizes at $20 \%$. 
Variance decomposition of model without investor sentiment. The variance decomposition of model without investor sentiment shows that the proportion of variable CPI that can be explained by M2 slowly increases from 1\% in the first period to $9 \%$ in the sixth period and stabilizes at $10 \%$ from the tenth period. The proportion of variable Q that can be explained by M2 slowly increases from 1\% in the first period to $6 \%$ in the fourth period, and then rapidly grows. It reaches $18 \%$ in the sixth period and remains stable at $18 \%$ afterwards.

\section{Conclusions}

\subsection{Analysis of impulse response results.}

Under the dramatic impacts of money supply, prices and output fluctuate sharply in short term. Positive and negative fluctuations appear alternatively. In the long run, the fluctuation gradually subsides. The responses of CPI and Q to M2 disturbance are similar in both models. But the degree of responses in the model without investor sentiment are smaller than those in the model with investor sentiment, indicating that investor sentiment can enhance the impact of monetary policy on the macro economy.

\subsection{Analysis of variance decomposition results.}

In both models, when money supply changes dramatically, the ratios of price and output which can be explained by money supply first gradually increase and then remain stable, indicating that the impacts of monetary policy on price and output has a time lag, but the effect is long-lasting. In addition, compared with the model without investor sentiment factors, the explanatory ratio of M2 to CPI and Q is higher in the model with investor sentiment factor, indicating that the explanatory power of monetary policy on price and output volatility can be enhanced when investor sentiment is taken into consideration.

\section{References}

[1] Q. Luo, Monetary Policy Transmission in China: Evidence from FAVAR Approach, Capital University of Economics and Business, 2015.

[2] Y. Zhou, an Empirical Study on the Influence of Investor Sentiment to Monetary Policy Transmission in China, Fudan University, 2012.

[3] A. Kurov., Investor sentiment and the stock market's reaction to monetary policy, J. Journal of Banking and Finance. 34 (2009).

[4] Y.Y. Sui, Empirical Study on the Influence of Monetary Policy on Investor Sentiment and Stock Market Volatility, Liaoning University, 2017. 